\title{
Article
}

\section{Minimal graphs for hamiltonian extension}

\author{
Christophe Picouleau \\ CEDRIC laboratory, Conservatoire National des Arts et Métiers, Paris, France.; christophe.picouleau@cnam.fr
}

Received: 16 January 2020; Accepted: 2 March 2020; Published: 8 March 2020.

\begin{abstract}
For every $n \geq 3$, we determine the minimum number of edges of graph with $n$ vertices such that for any non edge $x y$ there exits a hamiltonian cycle containing $x y$.
\end{abstract}

Keywords: 2-factor, hamiltonian cycle, hamiltonian path.

MSC: 26B25, 26A33, 26A51, 33E12.

\section{Introduction}

$\mathbf{F}$ or all graph theoretical terms and notations not defined here the reader is referred to [1]. We only consider simple finite loopless undirected graphs. For a graph $G=(V, E)$ with $|V|=n$ vertices, an edge is a pair of two connected vertices $x, y$, we denote it by $x y, x y \in E$; when two vertices $x, y$ are not connected this pair form the non-edge $x y, x y \notin E$. In $G$ a 2-factor is a subset of edges $F \subset E$ such that every vertex is incident to exactly two edges of $F$. Since $G$ is finite a 2 -factor consists of a collection of vertex disjoint cycles spanning the vertex set $V$. When the collection consists of an unique cycle the 2 -factor is connected, so it is a hamiltonian cycle.

We intend to determine, for any integer $n \geq 3$, a graph $G=(V, E), n=|V|$ with a minimum number of edges such that for every non-edge $x y$ it is always possible to include the non-edge $x y$ into a connected 2-factor, i.e., the graph $G_{x y}=(V, E \cup\{x y\})$ has a hamiltonian cycle $H, x y \in H$. In other words for any non-edge $x y$ of $G$ there exits a hamiltonian path between $x$ and $y$.

This problem is related to the minimal 2-factor extension studied in [2] in which the 2-factors are not necessary connected. It is also related to the problem of finding minimal graphs for non-edge extensions in the case of perfect matchings (1-factors) studied in [3]. Another problem of hamiltonian extension can be found in [4].

Definition 1. Let $G=(V, E)$ be a graph and $x y \notin E$ an non-edge. If $G_{x y}=(V, E \cup\{x y\})$ has a hamitonian cycle that contains $x y$ we shall say that $x y$ has been extended (to a connected 2-factor, to an hamiltonian cycle).

Definition 2. A graph $G=(V, E)$ is connected 2-factor expandable or hamiltonian expandable (shortly expandable) if every non-edge $x y \notin E$ can be extended.

Definition 3. An expandable graph $G=(V, E)$ with $|V|=n$ and a minimum number of edges is a minimum expandable graph. The size $|E|$ of its edge set is denoted by $\operatorname{Exp}_{h}(n)$.

The case where the 2-factor is not constrained to be hamiltonian is studied in [2]. In this context $\operatorname{Exp}_{2}(n)$ denotes the size of a minimum expandable graph with $n$ vertices. It follows that $\operatorname{Exp}_{h}(n) \geq \operatorname{Exp}_{2}(n)$.

We use the following notations. For $G=(V, E), N(v)$ is the set of neighbors of a vertex $v, \delta(G)$ is the minimum degree of a vertex. A vertex with exactly $k$ neighbors is a $k$-vertex. When $P=v_{i}, \ldots, v_{j}$ is a sequence of vertices that corresponds to a path in $G$, we denote by $\bar{P}=v_{j}, \ldots, v_{i}$ its mirror sequence (both sequences correspond to the same path).

We state our result. 
Theorem 1. The minimum size of a connected 2-factor expandable graph is:

$$
\operatorname{Exp}_{h}(3)=2, \operatorname{Exp}_{h}(4)=4, \operatorname{Exp}_{h}(5)=6 ; \operatorname{Exp}_{h}(n)=\left\lceil\frac{3}{2} n\right\rceil, n \geq 6
$$

Proof. For $n \geq 3$ we have $\operatorname{Exp}_{h}(n) \geq \operatorname{Exp}_{2}(n)$.

In [2] it is proved that the three graphs given by Figure 1 are minimum for 2-factor extension. They are also minimum expandable for connected 2-factor extension.
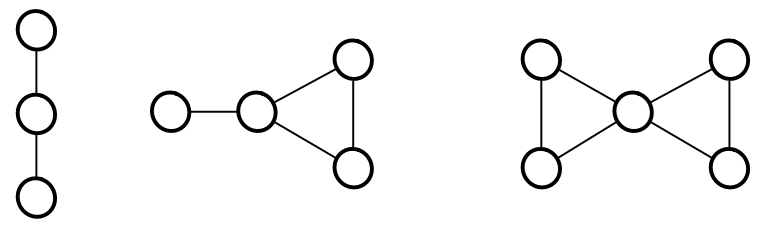

Figure 1. $P_{3}$, the paw, the butterfly.

Now let $n \geq 6$. From [2] we know the following when $G$ a minimum expandable graph for the 2 -factor extension:

- $G$ is connected;

- if $\delta(G)=1$ then $\operatorname{Exp}_{2}(n) \geq \frac{3}{2} n$;

- for $n \geq 7$, if $u, v$ are two 2-vertices such that $N(u) \cap N(v) \neq \varnothing$ then $\operatorname{Exp}_{2}(n) \geq \frac{3}{2} n$;

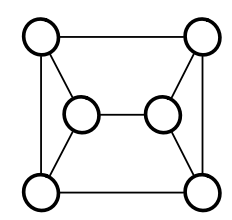

Figure 2. A minimum hamiltonian expandable graph with 6 vertices.

The graph given by Figure 2 is minimum for 2-factor extension (see [2]). One can check that it is expandable for connected 2-factor extension. So we have $\operatorname{Exp}_{h}(6)=9=\frac{3}{2} n$.

Suppose that $G$ is a minimum expandable graph with $n \geq 7$ and $\delta(G)=2$. Let $v \in V$ with $d(v)=2$, $N(v)=\left\{u_{1}, u_{2}\right\}$. If $u_{1} u_{2} \notin E$ then $u_{1} u_{2}$ cannot be expanded into a hamiltonian cycle. So $u_{1} u_{2} \in E$. If $d\left(u_{1}\right)=2$ then $u_{2} \in N\left(u_{1}\right) \cap N(v)$ and $\operatorname{Exp}_{h}(n) \geq \frac{3}{2} n$. So from now one we may assume $d\left(u_{1}\right), d\left(u_{2}\right) \geq 3$. Suppose that $d\left(u_{1}\right)=d\left(u_{2}\right)=3$. Let $N\left(u_{1}\right)=\left\{v, u_{2}, v_{1}\right\}, N\left(u_{2}\right)=\left\{v, u_{1}, v_{2}\right\}$. If $v_{1} \neq v_{2}$ then $u_{1} v_{2}$ is not expandable. If $v_{1}=v_{2}$ then $v v_{1}$ is not expandable. From now we can suppose that $d\left(u_{1}\right) \geq 3, d\left(u_{2}\right) \geq 4$. Moreover $v$ is the unique 2-vertex in $N\left(u_{2}\right)$. It follows that every 2-vertex $u \in V$ can be matched with a distinct vertex $u_{2}$ with $d\left(u_{2}\right) \geq 4$. Then $\Sigma_{v \in V} d(v) \geq 3 n$ and thus $m \geq \frac{3}{2} n$.

When $\delta(G) \geq 3$ we have $\Sigma_{v \in V} d(v) \geq 3 n$. Thus for any expandable graph we have $|E|=m \geq \frac{3}{2} n, n \geq 7$.

For any even integer $n \geq 8$ we define the graph $G_{n}=(V, E)$ as follows. Let $n=2 p, V=A \cup B$ where $A=\left\{a_{1}, \ldots, a_{p}\right\}$ and $B=\left\{b_{1}, \ldots, b_{p}\right\} . A$ (resp. $B$ ) induces the cycle $C_{A}=\left(A, E_{A}\right)$ with $E_{A}=$ $\left\{a_{1} a_{2}, a_{2} a_{3}, \ldots, a_{p} a_{1}\right\}$ (resp. $C_{B}=\left(B, E_{B}\right)$ with $E_{B}=\left\{b_{1} b_{2}, b_{2} b_{3}, \ldots, b_{p} b_{1}\right\}$. Now $E=E_{A} \cup E_{B} \cup E_{C}$ with $E_{C}=\left\{a_{2} b_{2}, a_{3} b_{3}, \ldots, a_{p-1} b_{p-1}, a_{1} b_{p}, a_{p} b_{1}\right\}$. Note that $G_{n}$ is cubic so $m=\frac{3}{2} n$. (see $G_{10}$ in Fig. 3)

We show that $G_{n}$ is expandable. First we consider a non-edge $a_{i} a_{j}, p \geq j>i \geq 1$. Note that the case of a non-edge $b_{i} b_{j}$ is analogous. We have $j \geq i+2$ and since $a_{1} a_{p} \in E$ from symmetry we can suppose that $j<p$. Let $P=a_{j}, a_{j-1}, \ldots, a_{i+1}, b_{i+1}, b_{i+2}, \ldots, b_{j+1}, a_{j+1}, a_{j+2}, b_{j+2}, \ldots, c_{j}$ where $c_{j}$ is either $a_{p}$ or $b_{p}$ and let $Q=a_{i}, b_{i}, b_{i-1}, a_{i-1}, \ldots, c_{i}$ where $c_{i}$ is either $a_{1}$ or $b_{1}$. From $P$ and $Q$ one can obtain an hamiltonian cycle containing $a_{i} b_{j}$ whatever $c_{i}$ and $c_{j}$ are.

Now we consider a non-edge $a_{i} b_{j}$. Without loss of generality we assume $j \geq i$. Suppose first that $j=i$, so either $i=1$ or $i=p$. Without loss of generality we assume $i=j=1: a_{1}, b_{p}, b_{p-1}, \ldots, b_{2}, a_{2}, a_{3}, \ldots, a_{p}, b_{1}, a_{1}$ is an 

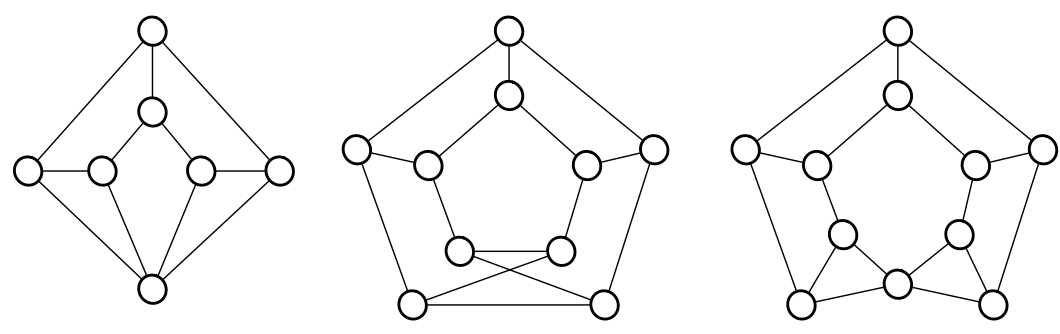

Figure 3. The graphs $G_{7}, G_{10}, G_{11}$, from the left to the right.

hamiltonian cycle. Now assume that $j>i$ : Let $P_{j}=b_{j}, b_{j-1}, \ldots, b_{i+1}, a_{i+1}, a_{i+2}, \ldots, a_{j+1}, b_{j+1}, b_{j+2}, a_{j+2}, \ldots, c_{p}$ where either $c_{p}=a_{p}$ or $c_{p}=b_{p}, P_{i}=a_{i}, b_{i}, b_{i-1}, a_{i-1}, a_{i-2}, \ldots, c_{1}$ where either $c_{1}=a_{1}$ or $c_{1}=b_{1}$. If $c_{p}=a_{p}$ and $c_{1}=a_{1}$ then $P_{j}, b_{1}, b_{p}, P_{i}, a_{j}$ is an hamiltonian cycle. If $c_{p}=a_{p}$ and $c_{1}=b_{1}$ then $P_{j}, a_{1}, b_{p}, P_{i}, a_{j}$ is an hamiltonian cycle. The two other cases are symmetric.

For any odd integer $n=2 p+1 \geq 7$ we define the graph $G_{n}=(V, E)$ as follows. We set $V=A \cup B \cup\left\{v_{n}\right\}$ where $A=\left\{a_{1}, \ldots, a_{p}\right\}$ and $B=\left\{b_{1}, \ldots, b_{p}\right\} . A \cup\left\{v_{n}\right\}$ (resp. $\left.B \cup\left\{v_{n}\right\}\right)$ induces the cycle $C_{A}=\left(A \cup\left\{v_{n}\right\}, E_{A}\right)$ with $E_{A}=\left\{a_{1} a_{2}, a_{2} a_{3}, \ldots, a_{p} v_{n}, v_{n} a_{1}\right\}$ (resp. $C_{B}=\left(B \cup\left\{v_{n}\right\}, E_{B}\right)$ with $E_{B}=\left\{b_{1} b_{2}, b_{2} b_{3}, \ldots, b_{p} v_{n}, v_{n} b_{1}\right\}$. Now $E=E_{A} \cup E_{B} \cup E_{C}$ with $E_{C}=\left\{a_{i} b_{i} \mid 1 \leq i \leq p\right\} \cup\left\{a_{1} v_{n}, b_{1} v_{n}, a_{p} v_{n}, b_{p} v_{n}\right\}$. Note that $m=\left\lceil\frac{3}{2} n\right\rceil$. (see $G_{7}$ and $G_{11}$ in Figure 3)

We show that $G_{n}$ is expandable. First, we consider a non-edge $a_{i} a_{j}, p \geq j>i \geq 1$ (the case of a non-edge $b_{i} b_{j}$ is analogous). $a_{i}, a_{i+1}, \ldots, a_{j-1}, b_{j-1}, b_{j-2}, b_{j-3}, \ldots, b_{i}, b_{i-1}, a_{i-1}, a_{i-2}, b_{i-2}, \ldots, v_{n}, c_{p}, d_{p}, d_{p-1}, c_{p-1}, \ldots, c_{j}, d_{j}$, where $d_{j}=a_{j}$ and for any $k, j \leq k \leq p$, the ordered pairs $c_{k}, d_{k}$ correspond to either $a_{k}, b_{k}$ or $b_{k}, a_{k}$, is an hamiltonian cycle. Second, let a non-edge $a_{i} b_{j}, p \geq j>i \geq 1$. We use the same construction as above taking $d_{j}=b_{j}$.

Acknowledgments: The author express its gratitude to Dominique de Werra for its constructive comments and remarks, which helped to improve the writing of this paper.

Conflicts of Interest: "The author declare no conflict of interest."

\section{References}

[1] Bondy, J.A., Murty, U. S. R. (2008). Graph Theory. Springer.

[2] Costa, M. C., de Werra, D., \& Picouleau, C. (2020). Minimal graphs for 2-factor extension. Discrete Applied Mathematics, accepted for publication.

[3] Costa, M. C., de Werra, D., \& Picouleau, C. (2018). Minimal graphs for matching extensions. Discrete Applied Mathematics, 234, 47-55.

[4] Zhang, P. (2016). Hamiltonian Extension. In Graph Theory (pp. 17-30). Springer, Cham.

(C) 2020 by the authors; licensee PSRP, Lahore, Pakistan. This article is an open access article distributed under the terms and conditions of the Creative Commons Attribution (CC-BY) license (http://creativecommons.org/licenses/by/4.0/). 